\title{
Dijet azimuthal distributions and initial-state parton showers
}

\author{
F. Hautmann ${ }^{1}$ and H. Jung ${ }^{2}$ \\ 1 - Oxford University, Theoretical Physics Department \\ Oxford OX1 3NP, UK \\ 2 - Deutsches Elektronen Synchrotron \\ Hamburg D-22603, Germany
}

\begin{abstract}
We investigate angular correlations in multi-jet final states at high-energy colliders and discuss their sensitivity to initial-state showering effects, including QCD coherence and corrections to collinear ordering 1 .
\end{abstract}

Presented at the Workshop DIS08, University College London, April 2008

Events with multiple hadronic jets are central to many aspects of the LHC physics program and their analysis will require realistic Monte Carlo simulations. See e.g. 22. In a multi-jet event the correlation in the azimuthal angle $\Delta \phi$, defined to be between the two hardest jets, provides a useful measurement, sensitive to how well QCD multiple-radiation effects are described, and has been used to tune shower Monte Carlo event generators 3 . The Tevatron $\Delta \phi$ measurements 4 admit a reasonable description by Monte Carlo, see Herwig and Pythia results in Fig. 1. 4. In particular the data are sensitive to initial-state showering parameters and have been used for re-tuning of these parameters in PyтніA [3. On the other hand, the HERA $\Delta \phi$ measurements [5, 6] are not well described by the standard Herwig and Pythia Monte Carlo showers in most of the data kinematic range (see below).

At the LHC, measurements of $\Delta \phi$ distributions in multi-jet events may become accessible relatively early. Such complex hadronic final states at LHC energies are potentially sensitive to corrections to the collinear ordering implemented in standard parton showers [7]. In particular, for jets of given $E_{T}$ the partonic momentum fraction $x$ is reduced as the energy increases, and angular correlations probe coherence effects in the spacelike branching [8], associated with non-collinear radiation at $x \ll 1$ and not included in HeRWIG or PYTHIA.

Monte Carlo generators designed to take these effects into account are based (see e.g. 9, 10. and early studies in [11) on transverse-momentum dependent parton distributions and matrix elements, defined via high-energy factorization [12]. General formulations for these distributions in initial-state showers are studied in [13.

Ref. [8] investigates the effects of corrections to collinear-ordered showers on correlations in multi-jet final states, using the precise ep measurements [6] that have recently become available. These measurements are characterized by large phase space available for jet production and by small $x$ kinematics, potentially relevant for extrapolation of initial-state showering effects to the LHC. In Fig. 2 we report results 8 for the azimuthal $\Delta \phi$ distribution in two-jet and three-jet cross sections. In Fig. 3 we give results for the $\Sigma p_{t}$ and $\Delta p_{t}$ distributions [6, 8, measuring the transverse-momentum imbalance between the leading jets.

These results show that the shape of the distributions is different for HERwIG and for the $\mathrm{k}_{\perp}$-shower Monte Carlo CASCADE 14, with the largest differences occurring at small $\Delta \phi$ and small $\Delta p_{t}$, where the two highest $E_{T}$ jets are far from back to back and one has

DIS 2008 


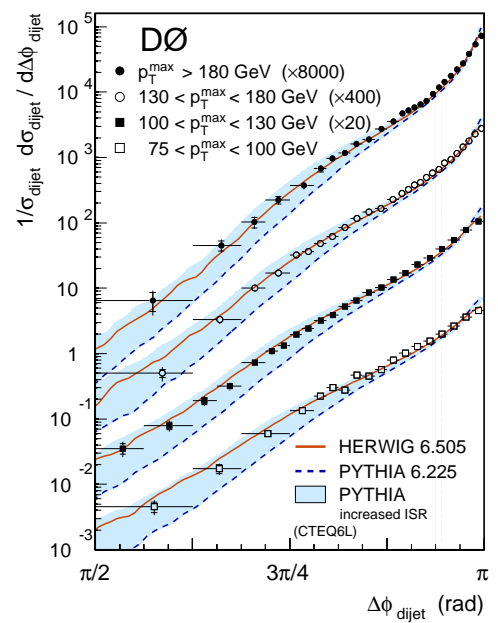

Figure 1: Dijet azimuthal correlations measured by D0 along with the HeRwIG and PyTHIA results [4.

effectively three hard, well-separated jets. Ref. [8] also analyzes the angular distribution of the third jet and finds significant contributions from regions where the transverse momenta in the initial state shower are not ordered. The description of the measurement by the $\mathrm{k}_{\perp}$-shower is good, whereas the collinear-based HERwIG shower is not sufficient to describe it.
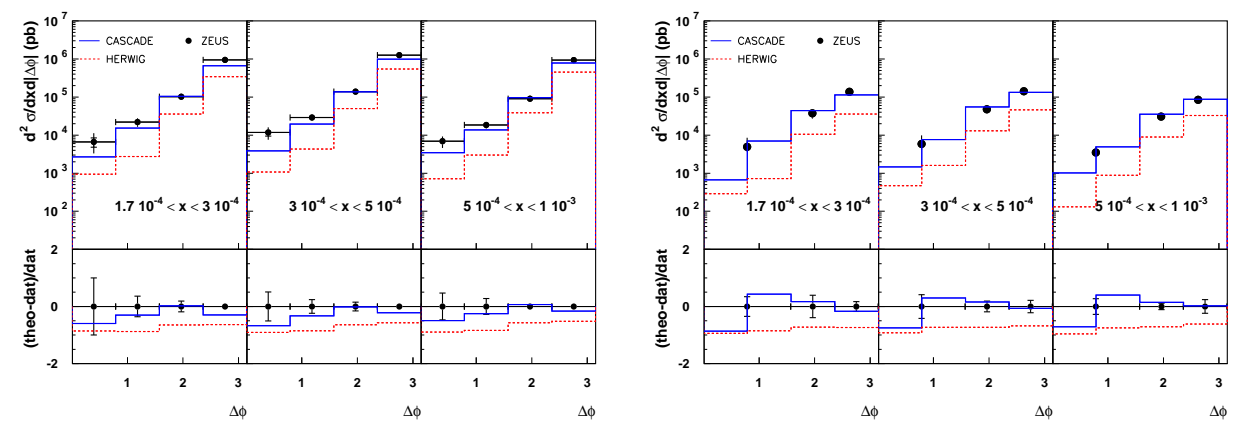

Figure 2: Azimuthal correlations $\left[\underline{8}\right.$ by the $\mathrm{k}_{\perp}$-shower CASCADE and by HeRwiG compared with the ep data [6]: (left) two-jet cross section; (right) three-jet cross section.

The physical picture underlying the $\mathrm{k}_{\perp}$-shower method involves both transverse momentum dependent pdfs and matrix elements [7. The angular and momentum correlations of Figs. 2]3 are found [8, 15] to be sensitive in particular to the large- $\mathrm{k}_{\perp}$ tail in the hard matrix elements [12. More detailed studies of these off-shell contributions are currently underway, including comparisons with results of next-to-leading order (NLO) event generators, see single-jet and di-jet distributions in Fig. 4. Here we see in particular that the dijet $p_{t}$ spectrum at high $p_{t}$ is close for the NLO calculation and the $\mathrm{k}_{\perp}$-shower (at low $p_{t}$ we see the effect of the Sudakov form factor in the shower). Ref. [8] illustrates that the collinear 
approximation to the matrix element does not describe the shape of the angular distribution at small $\Delta \phi$. We note that the inclusion of the perturbatively computed high- $\mathrm{k}_{\perp}$ correction distinguishes the calculation $[8$, of multi-jet cross sections from other shower approaches (see e.g. [16]) that include transverse momentum dependence in the pdfs but not in the matrix elements.
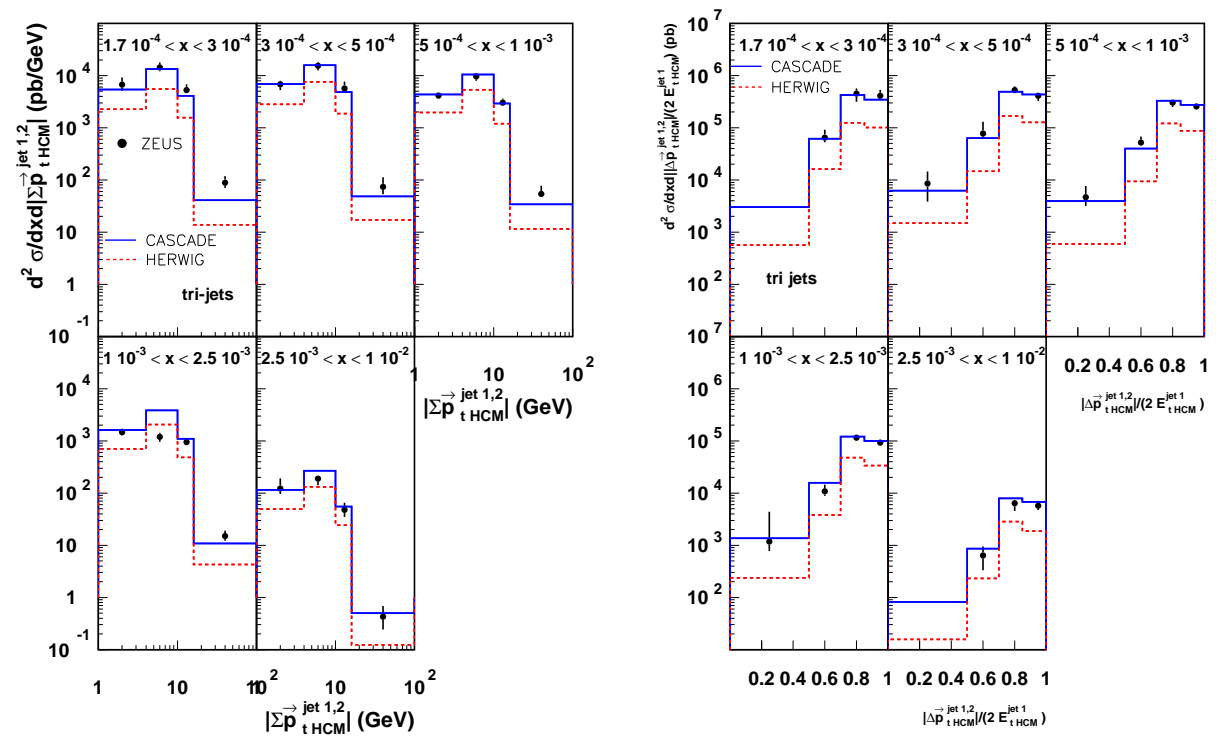

Figure 3: Transverse momentum correlations 8 by the $\mathrm{k}_{\perp}$-shower CASCADE and by HeRwIG compared with the 3 -jet data [6]. The variables $\Sigma p_{t}$ (left) and $\Delta p_{t}$ (right) are as defined in [6, 8].

It is worth emphasizing that the coherence effects in the angular distributions computed above are associated with multi-gluon radiation terms to the initial-state shower that become non-negligible at high energy (small $x$ ) and small $\Delta \phi$. These can be incorporated using the formulation at fixed transverse momentum. Near the back-to-back region of large $\Delta \phi$ [17, corrections due to mixed Coulomb/radiative terms may also become important and affect the basic picture: see recent studies in [18]. See also [19] for a related discussion of Coulomb contributions. More general issues on unintegrated pdfs in parton showers are discussed in [7, 13, 20. Applications to semi-inclusive processes and spin asymmetries are reviewed in [21].

Besides jet final states, the corrections to collinear-ordered showers discussed in this article will also be relevant to heavy particle production [10, 12, 22, including phenomenological studies of small- $x$ broadening in $\mathrm{W}$ and $\mathrm{Z} p_{\perp}$ distributions [23, kinematical relations of DIS event shapes with Drell-Yan production 24, heavy flavor production. First results on top-antitop pair production at the LHC may be found in the first paper of reference [7. 

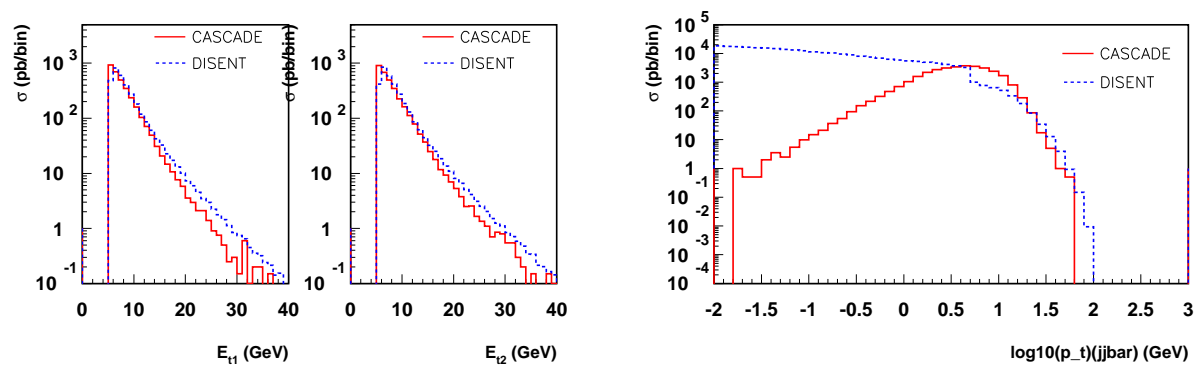

Figure 4: Comparison of the $\mathrm{k}_{\perp}$-shower CASCADE with the NLO di-jet calculation DisENT: (left) single-jet distributions; (right) di-jet distributions.

\section{References}

[1] Slides: http: //indico. cern. ch/contributionDisplay py? contribId=210\&sessionId=13\&conf Id=24657

[2] J. Alwall et al., Eur. Phys. J. C 53 (2008) 473 arXiv:0706.2569 [hep-ph]].

[3] M.G. Albrow et al. [TeV4LHC QCD Working Group], arXiv:hep-ph/0610012].

[4] V.M. Abazov et al. [D0 Collaboration], Phys. Rev. Lett. 94 (2005) 221801 arXiv:hep-ex/0409040.

[5] M. Hansson [H1 Collaboration], in Proceedings of DIS2006 (Tsukuba, April 2006), p. 539; A. Aktas et al. [H1 Collaboration], Eur. Phys. J. C 33 (2004) 477 arXiv:hep-ex/0310019].

[6] S. Chekanov et al. [ZEUS Collaboration], Nucl. Phys. B 786 (2007) 152 [arXiv:0705.1931 [hep-ex]].

[7] F. Hautmann and H. Jung, arXiv:0808.0873 [hep-ph]; arXiv:0712.0568 [hep-ph].

[8] F. Hautmann and H. Jung, arXiv:0805.1049 [hep-ph].

[9] J.R. Andersen et al., Eur. Phys. J. C48 (2006) 53 arXiv:hep-ph/0604189.

[10] H. Jung, Mod. Phys. Lett. A 19 (2004) 1 arXiv:hep-ph/0311249.

[11] B.R. Webber, hep-ph/9510283 G. Marchesini and B.R. Webber, Nucl. Phys. B386 (1992) 215.

[12] S. Catani, M. Ciafaloni and F. Hautmann, Phys. Lett. B 242 (1990) 97; Nucl. Phys. B366 (1991) 135; Phys. Lett. B 307 (1993) 147; S. Catani and F. Hautmann, Nucl. Phys. B427 (1994) 475.

[13] J.C. Collins and X. Zu, JHEP 0503 (2005) 059; T.C. Rogers, arXiv:0807.2430 [hep-ph]; arXiv:0712.1195 [hep-ph].

[14] H. Jung, Comput. Phys. Commun. 143 (2002) 100 arXiv:hep-ph/0109102.

[15] F. Hautmann and H. Jung, arXiv:0804.1746 [hep-ph], in Proceedings of the 8th International Symposium on Radiative Corrections RADCOR2007 (Florence, October 2007).

[16] S. Höche, F. Krauss and T. Teubner, arXiv:0705.4577 [hep-ph].

[17] A. Banfi, M. Dasgupta and Y. Delenda, arXiv:0804.3786 [hep-ph]; Y. Delenda, arXiv:0706.2172.

[18] J.C. Collins, arXiv:0708.4410 [hep-ph]; W. Vogelsang and F. Yuan, arXiv:0708.4398 [hep-ph].

[19] M.H. Seymour, arXiv:0710.2733 [hep-ph].

[20] F. Hautmann, Phys. Lett. B 655 (2007) 26 arXiv:hep-ph/0702196; arXiv:0708.1319 [hep-ph]; J.C. Collins and F. Hautmann, JHEP 0103 (2001) 016; Phys. Lett. B 472 (2000) 129.

[21] P.J. Mulders, arXiv:0806.1134 [hep-ph]; U. D'Alesio and F. Murgia, arXiv:0712.4328 [hep-ph].

[22] F. Hautmann, Phys. Lett. B 535 (2002) 159.

[23] S. Berge, P.M. Nadolsky, F.I. Olness and C.P. Yuan, hep-ph/0508215

[24] M. Dasgupta and Y. Delenda, JHEP 0608 (2006) 080. 